\title{
О МЕТОДАХ ОЦЕНКИ СТРАТЕГИИ РАЗВИТИЯ СОЦИАЛЬНОЙ СФЕРЫ РЕСУРСНОГО РЕГИОНА
}

\author{
С. М. Лавлинский ${ }^{\star}$, Л. Л. Яковлева ${ }^{\star *}$ \\ ${ }^{\star}$ Институт математики имени С. Л. Соболева СО РАН, \\ **Забайкальский государственный университет
}

Поступила в редакцию 12.02.2018 г.

\begin{abstract}
Аннотация. В статье предложен подход к разработке модельного инструментария, позволяющего оценить последствия реализации конкретного индикативного плана развития социальной сферы региона, определяющего динамику показателей уровня жизни населения. Возможности подхода иллюстрируются на примере Забайкальского края, для которого оцениваются перспективы реализации Стратегии развития Забайкальского края до 2030 года. В модели выделены демографический блок, блок бюджетного финансирования, блок социальной сферы и блок домохозяйств. Механизм формирования расходной части бюджета, направляемого на нужды отраслей социальной сферы, представляет собой алгоритм принятия решения, основу которого составляют прогнозы доходов и потребностей отраслей в совокупности с процедурами распределения по приоритетам, характеризующим сравнительную важность отдельных расходных статей в ситуации бюджетного дефицита.

Предлагаемый подход позволяет системно подойти к вопросам планирования отдельных отраслей социальной сферы и более корректного, чем это можно наблюдать на практике, способа программирования развития социальной сферы региона в целом.

Ключевые слова: индикаторы уровня жизни, сценарное прогнозирование, алгоритм формирования бюджета, оценка стратегических планов.

Annotation. The article suggests an approach to the development of modeling tools for assessing the consequences of implementing some specific region's social sphere development indicative plan, which determines the dynamics of the population's standard of living indicators. The possibilities of the approach are illustrated by the example of the Trans-Baikal Territory, for which the prospects of the "Strategy for the Development of the Trans-Baikal Territory up to 2030" implementation are estimated. Within the model, the demographic, the budget financing, the social and the household blocks were marked out. The mechanism of the formation of the expenditure part of the budget directed to the needs of social sectors is a decision-making algorithm based on forecasts of the incomes and needs of the industries in conjunction with the priority distribution procedures that characterize the comparative importance of particular expenditure items in the current year fiscal deficit situation.

The proposed approach allows us to plan the particular branches of the social sphere more systematically and a more correct way of programming the region social sector development as a whole.
\end{abstract}

Keywords: standard of living indicators, scenario prognostication, budget forming algorithm, strategic plans estimation.

В большинстве ресурсных регионов России основной проблемой стратегического планирования является разработка программы освоения природно-ресурсного потенциала, на долгосрочном горизонте обеспечивающей достойный уровень жизни населению территории. В процессе стратегического планиро-

() Лавлинский С. М., Яковлева Л. Л., 2018 вания здесь на первый план выходят критерии результативности управления и требование достижения заявленных результатов в четко установленные сроки и в пределах выделенных ресурсов. Это возможно лишь на основе повышения эффективности бюджетных расходов, связывая воедино понятные обществу социально значимые проекты и затраченные на них средства бюджета. В какой мере сегод- 


\section{С. М. Лавлинский, Л. Л. Яковлева}

няшний управленец готов к решению такого рода задач?

Если на кратко- и среднесрочных временных горизонтах уже существуют методические заделы, закрепленные в документах Минфина и Минэкономразвития, то для долгосрочных горизонтов даже на федеральном уровне многие стратегические решения представляются не в полной мере обоснованными, прежде всего, с точки зрения доказательной оценки их результативности. Для значительной части опубликованных стратегий и программ ресурсных регионов ([1], [2]) запланированная динамика ключевых индикаторов уровня жизни определяет напряженный характер принятого долгосрочного индикативного плана. Но сам характер документа и форма подачи материала оставляют без ответа ключевой вопрос о реализуемости, а, значит, и результативности принятого стратегического решения.

По мере доработки Стратегии-2030 [3] перед субъектами РФ в полный рост встанет проблема приведения региональных программных документов в соответствие с федеральными. И здесь важно расширить методологическое поле подходов к разработке долгосрочной стратегии регионального развития, дополнив уже устоявшиеся на качественном уровне содержательные приемы программирования модельным каркасом, придающим доказательную жесткость процедурам оценки результативности стратегического управленческого решения, прежде всего, с точки зрения достижимости заявленных целевых значений индикаторов уровня жизни населения. В идеале со временем здесь должна появиться информационная технология разработки стратегии и программы социально-экономического развития региона, имеющая в своей основе соответствующий региональным особенностям модельный инструментарий.

Статья посвящена описанию возможного подхода к построению такого инструментария для ресурсного региона. Во многих регионах этого типа стратегические программные документы не в полной мере учитывают специфику проблем, связанных с устойчивым раз- витием, и это сказывается, прежде всего, на механизмах оценки перспектив достижения заявленной цели.

\section{1. ТЕХНОЛОГИЯ ФОРМИРОВАНИЯ СТРАТЕГИИ}

Для региона стратегия и разрабатываемая на ее основе программа социально-экономического развития - основные плановые документы, призванные системно показать ожидаемые результаты развития территории, прежде всего, с точки зрения динамики уровня жизни населения. К сожалению, достаточно часто в сегодняшних региональных программных документах можно видеть неподкрепленные доказательным образом выводы об ожидаемой положительной динамике уровня жизни населения в результате реализации мероприятий, каждое из которых потенциально может привести к улучшениям отдельных индикаторов уровня жизни. Такого рода утверждения требуют серьезной доказательной базы, оценки уровня достижения стратегической цели развития в результате реализации и тщательного анализа возможностей ресурсного обеспечения запланированных мероприятий.

На сегодняшний день сложилась практика разработки стратегий и программ, основной акцент в которых сделан на «прорисовку» контуров проектных мероприятий и оценку соответствующей динамики производственных показателей. Вопросы о том, насколько изменится уровень жизни населения, и в какой мере сбалансирована программа развития с точки зрения обеспеченности ресурсами, как правило, не находят ответа. Необходимые прогнозные оценки сбалансированности бюджетных планов и доходов бюджета на протяжении всего рассматриваемого периода в таких программных документах чаще всего отсутствуют, а недостаточная согласованность отраслевых прогнозов между собой лишь усиливает потенциальную неустойчивость будущего развития и делает такие программы малоэффективными для практики регионального управления. 
Максимально остро задачи стратегического планирования стоят в ресурсных регионах - именно здесь общенациональная ориентация на развитие сырьевого сектора негативно воздействует на процесс формирования региональных пропорций и инициирует полный комплекс долгосрочных проблем, связанных с устойчивым развитием на фоне истощения природно-ресурсного потенциала. В этих условиях сложившаяся практика регионального программирования наносит ощутимый урон качеству и эффективности управления, концентрируя усилия на максимально эффектном способе подачи материала, вместо того, чтобы направить их на анализ и доказательство результативности предлагаемых мероприятий с точки зрения решения основной задачи, стоящей перед региональным руководством, - обеспечение ощутимого повышения уровня жизни граждан.

Для этого при разработке стратегии должны быть построены количественные оценки, как минимум, для технико-экономического сравнения базовых сценариев, размеров и состава ресурсов, а также предложений по финансированию за счет бюджетов. Необходима также серьезная инструментальная база, позволяющая не только оценить количественные параметры, но и спрогнозировать последствия реализации программы с точки зрения достижения заявленных целей.

До сих пор не решена фундаментальная задача создания информационной технологии формирования стратегии и программы социально-экономического развития ресурсного региона. Ее базовые элементы - компьютерные системы, математические модели, алгоритмы и методики - должны сформировать инструментальную систему поддержки процесса принятия управленческих решений, позволяющую строить эффективные сценарии достижения заявленных целей [4]. Стратегия и программа социально-экономического развития в рамках такой технологии текстуально закрепляет результаты многочисленных модельных экспериментов и потому обладает дополнительным запасом устойчивости к изменению внешних условий.
В центре информационной технологии должна быть модель объекта стратегического планирования. Мало того, что полноценная модель позволяет изначально рассмотреть широкое множество сценариев развития. В ситуации кризиса информационная технология обеспечивает оперативное проведение необходимых численных экспериментов, позволяющих учесть изменившееся состояние объекта управления и спрогнозировать последствия реализации представительного множества сценариев развития кризиса, а каждому из них противопоставить и модельно оценить свою систему компенсирующих мероприятий.

\section{2. БАЗОВАЯ ЗАДАЧА ИНДИКАТИВНОГО ПЛАНИРОВАНИЯ}

Для разработки стратегии и программы социально-экономического развития предлагается использовать подход, основанный на базовых идеях индикативного планирования. Ключевая роль здесь принадлежит формированию системы индикаторов, характеризующих состояние и развитие экономики, и государственному регулированию социально-экономических процессов, обеспечивающему достижение целевых значений индикаторов [5].

Индикативный план - комплексный документ, отражающий в виде конкретных значений индикаторов цели социально-экономического развития территории, намечаемые для их достижения мероприятия и средства реализации, а также необходимые материальные и финансовые ресурсы. Здесь основная проблема - разработка методики построения напряженного индикативного плана, определяющего реальные диапазоны основных индикаторов для территории и обеспечивающего достижение намеченных целей. Механизм построения индикативного плана должен обеспечивать сбалансированность намечаемых мероприятий по трудовым и материальным ресурсам, бюджетных планов и доходов бюджетов на протяжении всего рассматрива- 


\section{С. М. Лавлинский, Л. Л. Яковлева}

емого периода, а также возможность построения оценки уровня достижения стратегических целей развития в результате реализации индикативного плана.

По своей сути, стратегия - частично формализованный фрагмент долгосрочного индикативного плана, в рамках которого формулируются цели, определяются индикаторы целей и формируется набор индикаторов состояния социально-экономической сферы. Выбор значения целевых индикаторов и момента их достижения для ресурсного региона представляет собой весьма сложную задачу, решение которой требует, как минимум, корректной рентной оценки основных месторождений как источника ресурсов для повышения уровня жизни.

Программа, как прообраз регулятивной части индикативного плана, использует значения соответствующих индикаторов долгосрочного плана в качестве целевых ориентиров и фиксирует систему бюджетных приоритетов, формирующих объемы финансирования по отраслям и направлениям. В составе программы присутствуют также отдельные рычаги косвенного регулирования объемы бюджетного строительства, льготные кредиты, варианты тарифной политики, и т. п.

В реальном процессе стратегического планирования проблемное поле охватывает полный спектр управленческих задач от результативного бюджетирования до анализа сценариев внешних условий в прогнозном периоде. Здесь же мы ограничимся проблемой выбора напряженного, но достижимого значения целевых индикаторов развития социальной сферы, которая является по-настоящему «узким» местом регионального программирования для сырьевых территорий. Эту проблему предлагается решать в рамках подхода, основанного на использовании долгосрочного индикативного плана, полученного в результате решения следующей задачи [4].

Фиксируется горизонт прогноза $T$ и начальные (сегодняшние) значения индикаторов уровня жизни населения $U_{i}(0), i=1, \ldots, N I$.

Для заданных эталонных значений индикаторов уровня жизни $E_{i}, i=1, \ldots, N I$, и пла- нируемого момента выхода на этот уровень $T_{E}$ населением территории, необходимо разработать индикативный план социально-экономического развития:

- сбалансированный на всем прогнозном горизонте по трудовым, материальным и финансовым ресурсам;

- включающий сценарий освоения природно-ресурсной базы;

- обеспечивающий выполнение условий

$$
U_{i}(t) \geq E_{i}, i=1, \ldots, N I, T_{E} \leq t \leq T .
$$

Для разработки стратегии развития ресурсного региона предлагается подход, основанный на многократном решении задачи (1) для представительного множества сценариев внешних условий. Полученный в процессе решения набор индикативных планов, динамика каждой компоненты которых имеет количественные характеристики, позволяет содержательно интерпретировать обнаруженные тенденции и уже на этой основе формировать управленческое решение, обладающие свойством устойчивости к изменению внешних условий, сбалансированности и результативности в смысле (1).

\section{3. МОДЕЛЬНЫЙ ИНСТРУМЕНТАРИЙ}

Основной инструмент решения задачи (1) эволюционная модель ресурсного региона, позволяющая прогнозировать социально-экономические и экологические последствия принимаемых решений для фиксированного сценария внешних условий функционирования [2].

Такая модель имеет блочную структуру, настраиваемую на конкретную территорию, специальный инструментарий работы с объектами минерально-сырьевой базы и внутренние механизмы, позволяющие связать планы косвенного регулирования и изменения условий жизни населения. Рекуррентная вычислительная схема модели позволяет последовательно вычислять траекторию развития:

$$
\begin{aligned}
& Y(t+1)=F\left(Y(t), I P(t+1), S(t+1), E, T_{E}\right), \\
& \quad t=0, \ldots, T, \\
& Y(0)=Y^{0} .
\end{aligned}
$$


Здесь $t$ - номер года, $T$ - горизонт прогнозирования; $S$ - прогноз внешних условий функционирования, включающий гипотезы о динамике инфляции, ставки рефинансирования и курса национальной валюты, сценарий трансформации бюджетной и налоговой систем, рыночные прогнозы и т. п.; IP - регулятивная часть индикативного плана, включающаясценарийосвоения минерально-сырьевой базы, стратегию формирования расходной части территориального бюджета и т. п.

$Y(t)$ - набор социально-экономических показателей, характеризующих региональный воспроизводственный процесс и условия жизни населения территории. Компоненты вектора $Y$ определяют для соответствующего момента времени демографическую ситуацию, мощности отраслей производственной и социальной сферы, характеристики их развития, доходную и расходную части территориального бюджета, выплаченную зарплату по отраслям и т. п.; $E=\left\{E_{i}, i=1, \ldots, N I\right\}$ и $T_{E}$-целевые значения индикаторов уровня жизни и планируемый год их достижения.

Уровень жизни характеризуется в модели целым набором целевых индикаторов - уровнями доходов, накоплений и занятости населения, а также параметрами обеспеченности населения объектами социальной сферы. Среднедушевой доход, генерируемый на территории, играет важную роль в оценке уровня жизни, но, тем не менее, не является единственным параметром, его определяющим. Кроме доходов на уровень жизни населения существенно влияет то, насколько жители обеспечены услугами отраслей социальной сферы и сколько им приходится платить за их использование. В рамках данной модели мы обходимся сравнительно небольшим перечнем индикаторов уровня жизни, исключив из рассмотрения большую часть индикаторов качества самого населения (показатели уровня образованности, квалификации, физического здоровья, стабильности семьи, социальных патологий и т. п.).

Оператор $F$ системы формализован в виде совокупности блоков имитационных алгоритмов, описывающих процессы функционирования основных экономических агентов на территории, их взаимодействие, применяемые на практике процедуры принятия текущих производственных решений, возникающие в результате материальные и финансовые потоки всех видов, а также логику формирования индикаторов уровня жизни.

Экзогенно сформировав индикативный план IP и описав начальное состояние территории $Y(0)$, эксперт, с помощью рекуррентных уравнений модели, прямым счетом, не решая систему уравнений классическим образом, получает $\{Y(t), t=0, \ldots, T\}-$ траекторию развития региональной экономики, анализ которой позволяет сделать вывод о перспективах достижения цели в рамках исходного варианта индикативного плана при реализации фиксированного сценария внешних условий.

\section{4. ПРИМЕР ОЦЕНКИ СТРАТЕГИЯ РАЗВИТИЯ ЗАБАЙКАЛЬСКОГО КРАЯ ДО 2030 ГОДА}

Для иллюстрации возможностей предлагаемого подхода рассмотрим Стратегию развития Забайкальского края до 2030 года [6], в которой зафиксированы достаточно высокие целевые индикаторы уровня жизни, достижение которых планируется к концу периода. Так, уровень обеспеченности жильем должен вырасти с 22 кв. м. в 2016 году до 30 кв. м. в 2030 году, рост обеспеченности дошкольными учреждениями запланирован на уровне 50, а здравоохранения - 100 процентов. В какой мере обоснованы такие показатели индикативного плана развития Забайкалья в сложившихся реалиях дотационного малоосвоенного ресурсного региона? При каких условиях заявленные эталонные значения индикаторов уровня жизни в 2030 году могут быть достигнуты?

Для того, чтобы ответить на эти вопросы, была использована эволюционная модель ресурсного региона (2), позволяющая оценить последствия реализации конкретного индикативного плана развития социальной сферы с точки зрения динамики показателей уровня жизни на территории. Система регуляторов в модели построена как комплекс мер органов 


\section{С. М. Лавлинский, Л. Л. Яковлева}

власти, направленных на достижение целевых значений индикаторов развития. Основными регуляторами являются элементы бюджетной и тарифной политики. В этих условиях основная задача - прогноз динамики индикаторов, порождаемой фиксированной политикой финансирования социальных отраслей и формирования тарифов для населения, а также определение политики регулирования, при которой достигаются целевые значения индикаторов с учётом ограниченности финансовых, материальных и иных ресурсов.

В рамках модельного инструментария центральную роль здесь играют демографический блок, блок бюджетного финансирования, блок социальной сферы и блок домохозяйств.

В демографическом блоке модели используются уравнения передвижки возрастов, позволяющие определить динамику общей численности населения и отдельных групп реципиентов услуг отраслей школьного и дошкольного образования. В блоке домохозяйств имитируется процесс формирования доходов и расходов населения, стратифици- рованного по уровню доходов. Здесь используются алгоритмы, позволяющие построить платежеспособный спрос на услуги негосударственного сектора социальной сферы.

В блоке социальной сферы представлены государственный и негосударственный секторы, в рамках которых выделены ЖКХ, образование (школы и дошкольные учреждения), здравоохранение (больницы и поликлиники), культура и физическая культура. Здесь моделируется динамика мощностей в натуральных показателях с учётом процессов физической амортизации (выбытия мощностей), ввода новых мощностей и эксплуатационных затрат на функционирование отраслей. В государственном секторе финансирование осуществляется за счет бюджета и зависит от уровня заработной платы и тарифов, платежеспособный спрос в негосударственном секторе определяет динамику мощностей и объёмы платных услуг населению, предоставленных вне государственного сектора.

Процедура формирования плана капитальных вложений отрасли социальной сферы в государственном секторе основана на

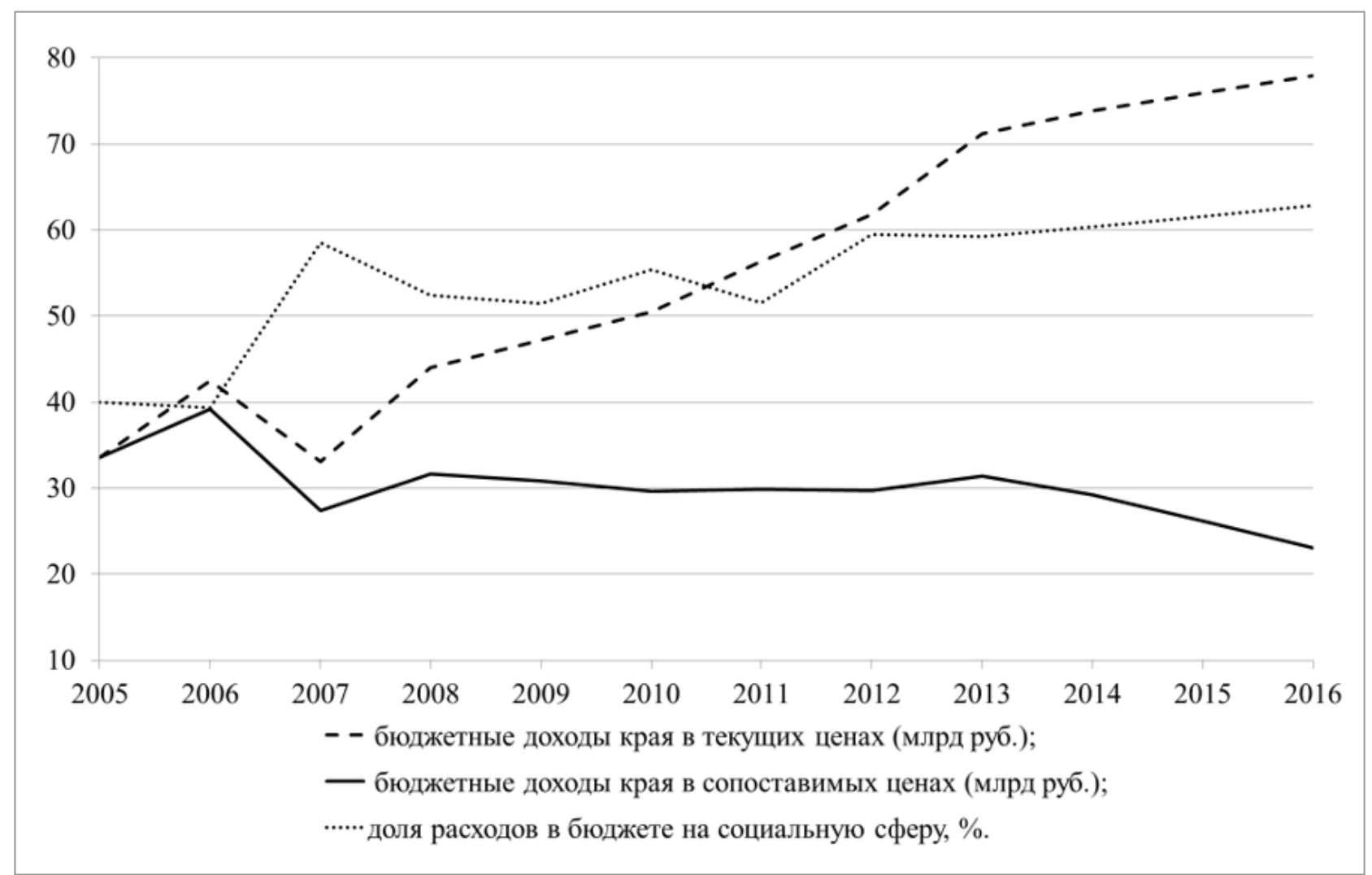

Рис. 1. Динамика доходов краевого бюджета и доли расходов на соииальную сферу 
сопоставлении текущего значения индикатора обеспеченности населения мощностями отрасли и уровня этого индикатора, предусмотренного индикативным планом. Необходимый объем нового строительства выбирается так, чтобы в следующем году выйти на запланированные значения индикаторов обеспеченности. Планы капитальных вложений и эксплуатационных затрат вкупе формируют заявку отрасли на бюджетное финансирование.

Основная задача, ежегодно решаемая в блоке бюджетного финансирования, заключается в распределении выделенных на социальную сферу ресурсов между отраслями таким образом, чтобы в конечном итоге выйти на показатели индикативного плана, определённые в стратегии развития и региональных целевых программах. При этом мы должны учесть инфляцию, индексацию тарифов и зарплат, а также объем негосударственных платных услуг и ввода жилья за счёт населения.

На рис. 1 представлена динамика основных характеристик бюджета Забайкальского края за 2005-2016 годы [7]. Она фиксирует тенденцию к снижению расходов бюджета в сопоставимых ценах, сопровождаемую ростом доли расходов на социальную сферу. Такая «социальная направленность» бюджета региона не является благом и приводит к тому, что в последние годы краевой бюджет является не бюджетом развития, а, скорее, бюджетом «выживания». На этом фоне заявленные эталонные значения индикаторов уровня жизни в Стратегии априори вызывает определенные сомнения в их реализуемости.

Численные эксперименты с моделью подтверждают эти сомнения. На рис. 2 представлена динамика уровня достижения целевых значений индикаторов обеспеченности в предположении, что наблюдаемые в ретроспективе тенденции развития края сохраняются до 2030 года (сценарий А). Этот сценарий предполагает, что темп падения бюджета в сопоставимых ценах составляет $1 \%$, а роста доли расходов бюджета на социальную сферу - $2 \%$. В рамках сценария А инфляция предполагается достаточно высокой (6-8 \%), а регулирование включает в себя индексацию заработной платы в государственном секторе социальной сферы на 2-4 \% ниже инфляции и тарифов на уровне инфляции. Итог сохранения негативных тенденций развития вполне закономерен и не обеспечивает достижения целевых показателей развития социальной сферы, зафиксированных в Стратегии развития Забайкалья (рис. 2, сценарий А).

Каковы должны быть условия, чтобы к 2030 году был выполнен заложенный в Стратегию индикативный план развития социальной сферы Забайкальского края?

Модель позволяет определить ключевые параметры таких условий, связанные, в первую очередь, с темпом роста бюджета. Зафиксировав сценарные условия В, предполагающие инфляцию на уровне $4 \%$ и основные элементы политики регулирования - темпы роста доли бюджета, направляемой в социальную сферу, а также стратегию индексации тарифов и зарплат на уровне инфляции, мы с помощью модели (2) можем каждому значению темпа роста бюджета сопоставить оценку уровня достижения эталонных показателей обеспеченности населения $\left(U_{i}(t) / E_{i}\right)$. Таким образом многократно решая обратную (1) задачу, выясняется, что для реализации целей Стратегии развития Забайкальского края до 2030 года необходим темп роста краевого бюджета на уровне 12,5 \% в сопоставимых ценах. Таких показателей формирования доходной части бюджета никогда не было в предшествующие годы, и представляется маловероятным их взрывной рост в долгосрочной перспективе, а значит и достижение заявленных в Стратегии целей.

\section{5. ОБСУЖДЕНИЕ ПОЛУЧЕННЫХ РЕЗУЛЬТАТОВ}

Анализ результатов экспериментов говорит о том, что использование модели позволяет оценить возможности достижения основных целей, заявленных в стратегии развития и связанных с формированием уровня жизни населения региона. Модель также может быть применена и для решения обратной задачи - задачи определения объёмов ресурсного обеспечения (в первую очередь финан- 

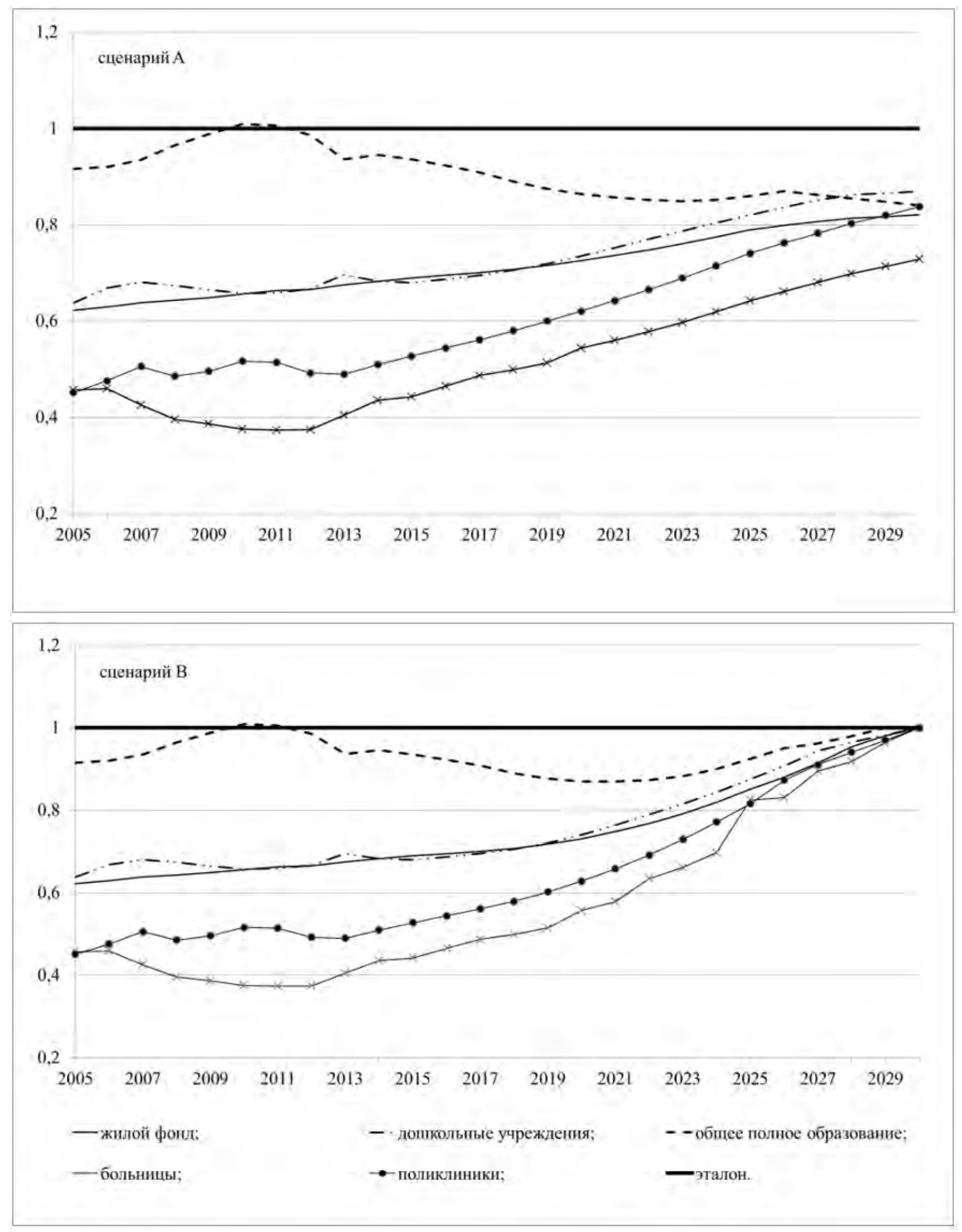

Рис. 2. Динамика уровня достижения целевых значений индикаторов обеспеченности 
сового), необходимого для выполнения индикативного плана.

В нашем случае, для достижения зафиксированных в Стратегии Забайкальского края высоких целевых показателей 2030 года требуется рост бюджета в 3 раза. Реализация инерционного сценария - сохранения исторически сложившихся тенденций величины дотаций из федерального бюджета порядка $18 \%$ - не позволяет достичь запланированных значений индикаторов уровня жизни.

Это лишний раз подтверждает высокий уровень недофинансирования региональных экономик - передав широкие полномочия, федеральный центр не в полной мере подкрепляет их ресурсами. Это приводит к стагнации и деградации и без того проблемных территорий, основной экономический потенциал которых сосредоточен в природно-ресурсной сфере, а уровень развития производственной инфраструктуры недостаточен для привлечения частных инвестиций.

Предлагаемый модельный инструментарий позволяет внести ясность в процедуру оценки стратегических планов развития региона. В региональных администрациях эта функция управления, как правило, не подкреплена соответствующими системами поддержки решений. Предлагаемый подход позволяет системно подойти к вопросам планирования отдельных отраслей социальной сферы и более корректного, чем это можно наблюдать на практике, способа программирования развития социальной сферы региона в целом.

Работа поддержана грантами РГНФ (проект № 16-02-00049) и РФФИ (проект № 16-0600046, расчёть произведень при поддержке гранта (проект № 16-02-00102)).

Лавлинский Сергей Михайлович - д-р техн. наук, ведущий научный сотрудник лаборатории математических моделей принятия решений института математики имени С. Л. Соболева СО РАН, г. Новосибирск.

E-mail: lavlin@math.nsc.ru

Яковлева Лидия Леонидовна - доцент кафедры прикладной информатики и математики Забайкальского государственного университета, г. Чита. E-mail: Lidia70@mail.ru

\section{СПИСОК ЛИТЕРАТУРЫ}

1. Стратегия экономического развития Тюменской области до 2020 года. - Режим доступа: http://www.urbanistika.ru/portfolio/ zodchestvo-2005-6/strategiya-tyumenskoyoblasti/TYuMEN_strategich.pdf (дата обращения 15.01.2018 г).

2. Стратегия социально-экономического развития Красноярского края на период до 2020 года. - Режим доступа: http:// economy.gov.ru/wps/wcm/connect/c9038c3349bc-4c42-b8bc-8269b763b21d/krasstrateg. $\mathrm{pdf} ? \mathrm{MOD}=\mathrm{AJPERES} \& \mathrm{CACHEID}=\mathrm{c} 9038 \mathrm{c} 33-$ 49bc-4c42-b8bc-8269b763b21d (дата обращения 15.01.2018 г).

3. Прогноз долгосрочного социально экономического развития российской федерации на период до 2030 года. - Режим доступа: http://static.government.ru/media/ files/41d457592e04b76338b7.pdf (дата обращения 15.01.2018 г).

4. Лавлинский С. М. Модели индикативного планирования социально-экономического развития ресурсного региона / С.М. Лавлинский. -Новосибирск. : Изд-во СОРАН, 2008. 247 c.

5. Цыбатов В. А. Моделирование экономического роста: [монография] / В. А. Цыбатов. - Самара. : Изд-во Самарского гос. экономического ун-та, 2006. - 359 с.

6. Стратегия развития Забайкальского края 2030. - Режим доступа: http://правительство.забайкальскийкрай.pф/blog/416.html (дата обращения: 20.12.2017).

7. Официальный сайт министерства финансов Забайкальского края. - Режим доступа: http://минфин.забайкальскийкрай.рф/ (дата обращения: 20.12.2017).

Lavlinskiy Sergey Mikhaylovich - Doctor of Technical Science, leading research fellow of the Mathematical Models of Decision Making Laboratory in Sobolev Institute of Mathematics, Novosibirsk.

E-mail: lavlin@math.nsc.ru

Yakovleva Lidiya Leonidovna - Associate Professor of the Applied Informatics and Mathematics Department in Transbaikal State University, Chita. E-mail: Lidia70@mail.ru 